\title{
IN-SITU MICROPLASMAS FOR RAPID DRY ETCHING OF SILICON
}

\author{
Chester G. Wilson and Yogesh B. Gianchandani* \\ Department of Electrical and Computer Engineering \\ University of Wisconsin, Madison
}

\begin{abstract}
This paper reports on the generation of spatially confined plasmas and their application to silicon etching. The etching is performed using $\mathrm{SF}_{6}$ gas and $\mathrm{DC}$ power applied between thin-film electrodes patterned on the silicon wafer to be etched. The typical operating pressure and power density are in the range of 1-20 Torr and 1-10 $\mathrm{W} / \mathrm{cm}^{2}$, respectively. The plasma confinement can be varied from $<100 \mu \mathrm{m}$ to $>1 \mathrm{~cm}$ by varying the electrode area, operating pressure, and power. High power densities can be achieved at moderate currents because the electrode areas are small. Etch rates of $4-17 \mu \mathrm{m} / \mathrm{min}$., which enable through-wafer etching and varying degrees of anisotropy, have been achieved. The etch rate increases with power density, whereas the etch rate per unit power density increases with operating pressure. Plasma resistance measurements and electric field modeling are used to provide an initial assessment of the microplasmas.
\end{abstract}

\section{INTRODUCTION}

Plasma processing is extensively utilized in semiconductor processing applications, and is the dominant silicon etching technique. Silicon etching is commonly performed in parallel plate reactors by applying RF power (typically at $13.56 \mathrm{MHz}$ ) between two electrodes placed several centimeters apart. The silicon wafer is located on the powered electrode for reactive ion etching. The operating pressure and power are in the range of 10-500 $\mathrm{mTorr}$ and $10-500 \mathrm{~mW} / \mathrm{cm}^{2}$, respectively. Since the plasma exists globally across the wafer, the etch is selectively masked by a thin film of photoresist, $\mathrm{SiO}_{2}$, or metal which is patterned on the wafer surface. More recently, fast anisotropic etches have been demonstrated by alternative plasma etchers utilizing electron cyclotron resonance (ECR) [1] and inductively coupled plasmas (ICP) [2]. All of these options, however, employ a single plasma that acts over the entire surface area of a wafer. Creating several different etch depths or profiles in a single die mandates the use of a like number of masking steps.

In this effort we report on silicon etching using in-situ microplasmas. The microplasmas are powered by two metal electrodes separated by a dielectric in a tri-layer stack on the wafer surface (Fig. 1). The power density is approximately $100 \times$ higher than in conventional plasmas. In addition, DC power is used to etch silicon substrates. This eliminates the need for matching impedance networks to associated with RF driven plasmas. Plasma confinement can be varied from a few tens of microns to more than a centimeter by changing operating conditions. The etch dimensions can also be confined to the openings in the mask, as with conventional etchers. Localized throughwafer etching has been demonstrated with this technique, and etch rates greater than $17 \mu \mathrm{m} / \mathrm{min}$. have been recorded using $\mathrm{SF}_{6}$.

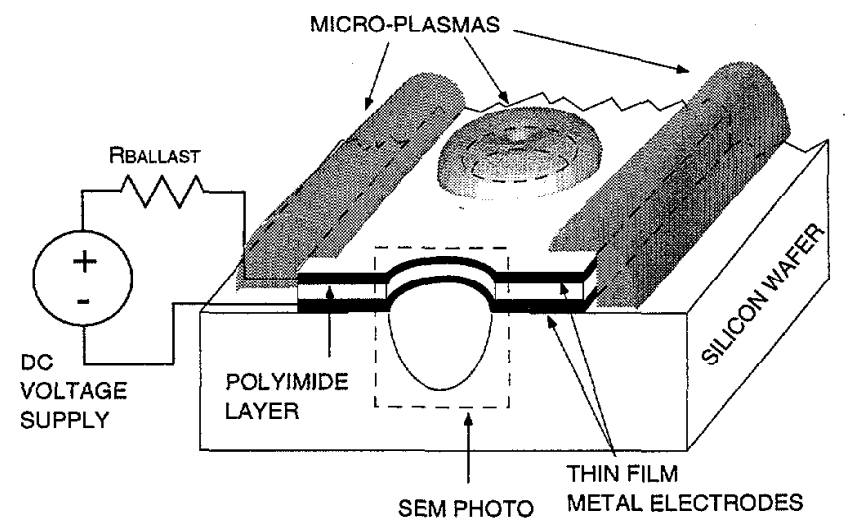

Fig. 1: Schematic illustrating the generation of an in-situ microplasma.

\section{EXPERIMENTAL RESULTS}

Test samples are fabricated by a two-mask process sequence. A metal-polyimide-metal electrode stack is deposited on the wafer. The first lithography mask is used to pattern the upper metal layer and the polyimide. The second mask is then used to pattern the lower metal layer and simultaneously re-pattern the upper metal as well. Metal layers that have been investigated include chrome, aluminum, titanium, and nickel. Following the microplasma etch, the electrode stack can be stripped by sacrificing the lower metal electrode in the appropriate wet etchant.

In the electrical set-up, the pads for the two in-situ electrodes are contacted by probes and connected to a DC power supply across a series ballast resistor, which provides a means to control the plasma current. The lower electrode is connected to the negative terminal of the supply, as shown in Fig. 1, in order to direct the positive ions in the plasma toward the Si substrate. Typical bias values range from 300-600 V, depending on the ambient gas used and target etch rate. The silicon is shielded from the electric field by the electrode stack itself. The relatively small

"Corresponding author: 1415 Engineering Drive, Madison, WI 53706-1691; Tel: (608) 262-2233, Fax: 262-1267, E-mail: yogesh@engr.wisc.edu 
electrode areas for in-situ microplasmas allow power densities in the range of $1-10 \mathrm{~W} / \mathrm{cm}^{2}$ without drawing high currents. The relatively large operating pressures of 1-20 Torr serve to spatially confine the plasma. This allows several microplasmas with different etch characteristics to operate simultaneously on a wafer.

The spatial self-confinement of microplasmas is shown in Fig. 2 as a glow that is localized to the in-situ electrodes. In this experiment, the ambient gas was $\mathrm{N}_{2}$, and the bias voltage was $-360 \mathrm{~V}$, which has opposite polarity to the bias normally used for etching. By varying the power and pressure the confinement can be changed from $<100$ $\mu \mathrm{m}$ to $>1 \mathrm{~cm}$.

Typical etch profiles achieved by microplasmas are shown in Figs. 3-5. In each of these cases the etch was performed though openings in the in-situ electrodes using a partial pressure of $\mathrm{SF}_{6}$. Figure 3(a) shows a $92 \mu \mathrm{m}$ deep etch through a circular opening of $150 \mu \mathrm{m}$ diameter, that was achicved in $20 \mathrm{~min}$. at 2.7 Torr, with a power density of $3.2 \mathrm{~W} / \mathrm{cm}^{2}$ averaged over the electrode area. The sidewall angle was $21^{\circ}$ off vertical. The electrode metal used in this case is aluminum. Figure 3(b) shows a $233 \mu \mathrm{m}$ deep etch that was achieved in $50 \mathrm{~min}$. using the same parameters. Figure 4 shows a $33 \mu \mathrm{m}$ deep etch through a $50 \mu \mathrm{m}$ diameter opening achieved in $3 \mathrm{~min}$. at 2.9 Torr and $7.3 \mathrm{~W} / \mathrm{cm}^{2}$. The sidewall angle is nearly vertical in certain locations of the profile. The electrode metal for this case was also aluminum. Figure 5 shows the cross-section of a $207 \mu \mathrm{m}$ deep etch through a $280 \mu \mathrm{m}$ wide, $2.2 \mathrm{~mm}$ long slit opening. The electrode metal used in this case was titanium. The etch was achieved in $24 \mathrm{~min}$. at 5.2 Torr and $6.8 \mathrm{~W} / \mathrm{cm}^{2}$. The sidewall angle of this etch is also nearly vertical in certain locations of the profile. The profiles shown in Figs. 3-5 indicate that varying degrees of anisotropy can be achieved by changing the operating. conditions of microplasmas.

The etch rate of $\mathrm{Si}$ in $\mathrm{SF}_{6}$ microplasmas was studied as a function of several operating parameters (Figs. 6-8). Figure 6 shows the dependence of the etch depth and the cumulative average etch rate on the duration of the etch. This etches were performed under two sets of conditions: case A used 2.7 Torr pressure, $1.6 \mathrm{~W} / \mathrm{cm}^{2}$ power density, and aluminum electrodes with circular openings of $350 \mu \mathrm{m}$ diamctcr; casc B used 5.2 Torr, $6.8 \mathrm{~W} / \mathrm{cm}^{2}$, and titanium electrodes with $280 \mu \mathrm{m}$ wide slit openings. Case A achieved etch rates of $4-7 \mu \mathrm{m} / \mathrm{min}$., whereas Case B achieved etch rates of 9-12 $\mu \mathrm{m} / \mathrm{min}$. In both cases the etch rate was significantly higher for the first few minutes, and then rapidly settled at a lower value that was stable for 50 minutes. Although the reasons for the higher initial etch rate remain to be determined, it is noteworthy that the electric field above the exposed $\mathrm{Si}$ is highest for the first few minutes of an etch, during the time that the etched depth is relatively small. This is shown as a modeling result in the next section.

Figure 6(a) also indicates that under the conditions of case $B$, it was possible to etch though a wafer in less than an hour. Through-wafer etches were routinely achieved with the use of titanium electrodes, which developed considerably less damage than aluminum from sputtcring in exposed regions of the cathode. This is consistent with trends seen in conventional etchers [3].

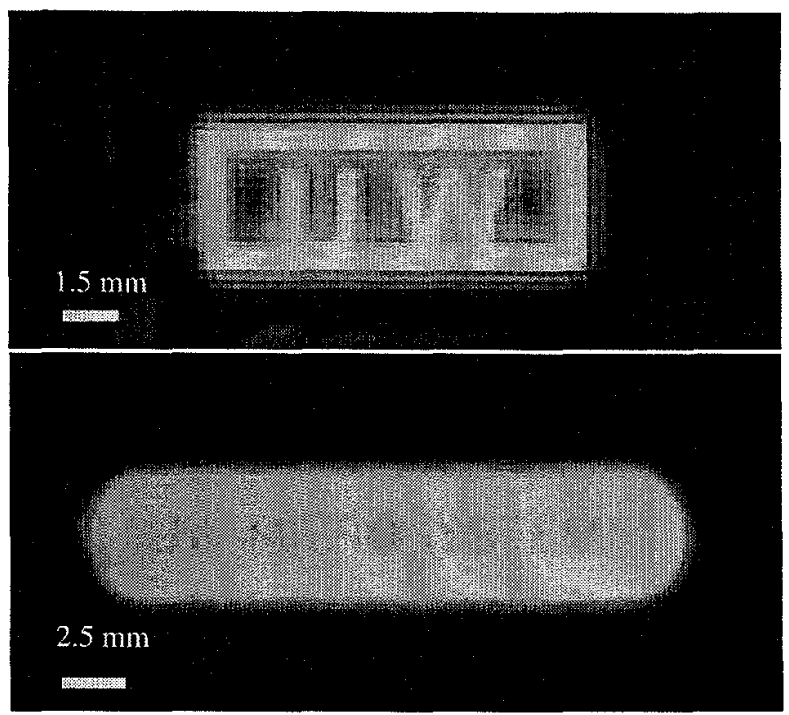

Fig. 2: Local confinement of $\mathrm{N}_{2}$ microplasmas at 1-20 Torr and $-360 \mathrm{~V} \mathrm{DC}$. (a-upper): Narrow electrodes with wide gaps; (b-lower): Wide electrodes with narrow gaps.
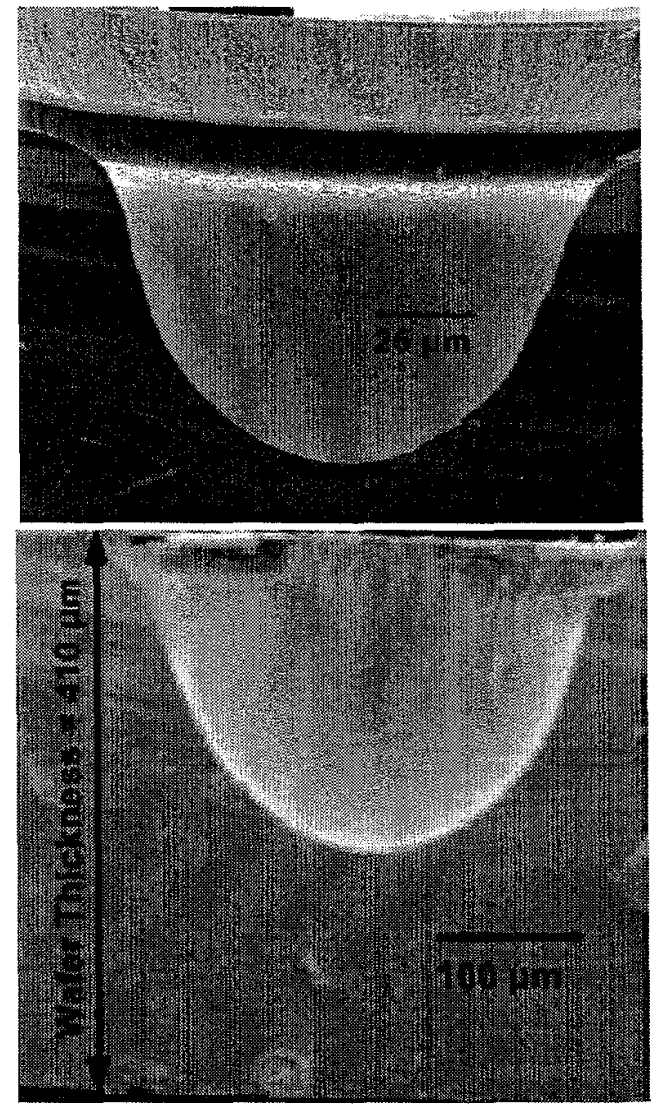

Fig. 3 (a-upper): A $92 \mu \mathrm{m}$ deep etch through a $150 \mu \mathrm{m} \emptyset$ circular opening (2.7 Torr, $3.2 \mathrm{~W} / \mathrm{cm}^{2}, 20 \mathrm{~min}$.). (b-lower): A $233 \mu \mathrm{m}$ deep etch $(50 \mathrm{~min}$.). 


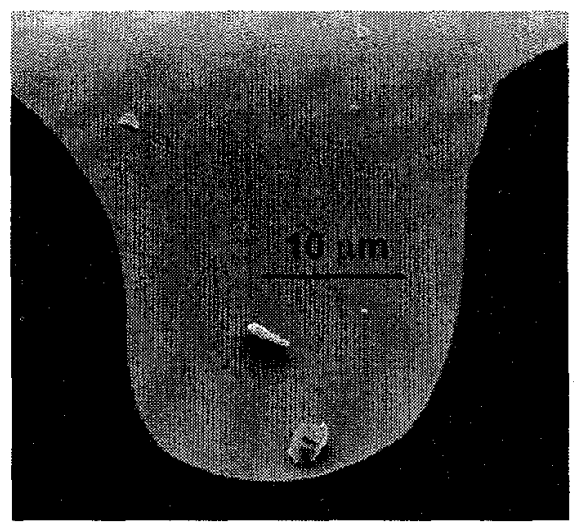

Fig 4: A $33 \mu \mathrm{m}$ deep etch through a $50 \mu \mathrm{m} \emptyset$ opening (2.9 Torr, $7.3 \mathrm{~W} / \mathrm{cm}^{2}, 3 \mathrm{~min}$.).

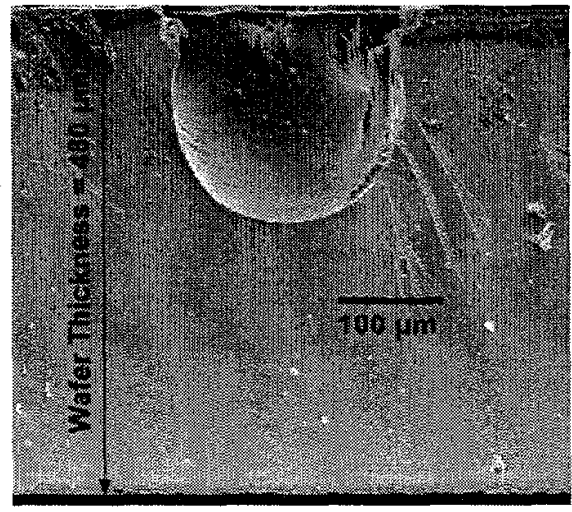

Fig 5: A $207 \mu \mathrm{m}$ deep etch through a $280 \mu \mathrm{m}$ wide slit opening (5.2 Torr, $6.8 \mathrm{~W} / \mathrm{cm}^{2}, 24 \mathrm{~min}$.).
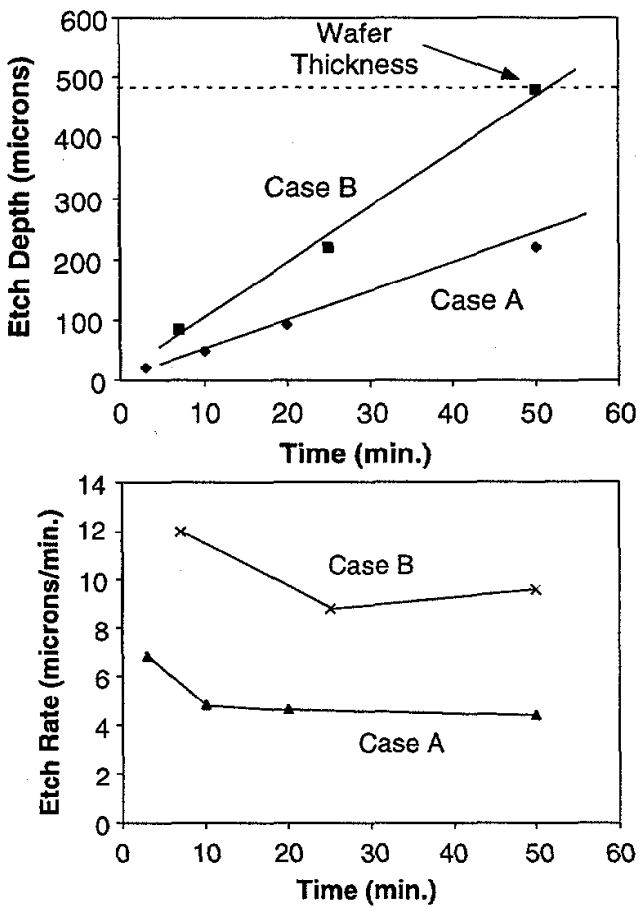

Fig 6 (a-upper): Etch progression for two cases described in the text. Through-wafer etches can be achieved in $<1$ hour. (b-lower): A cumulative average etch rate of $4-10 \mu \mathrm{m} / \mathrm{min}$. has been sustained, although initial values are higher.
Figure 7 shows the dependence of the etch rate on electrode power density. All the etches shown in this figure were performed for threc minutes at 2.7 Torr using aluminum electrodes with circular openings of $350 \mu \mathrm{m}$ diameter. It is clear that the etch rate increases linearly with power density within the operating regime explored. The highest etch rate achieved is $17.4 \mu \mathrm{m} / \mathrm{min}$. The relatively high etch rates are facilitated in part by the high power densities. Since the electrode area for microplasmas is generally $<<1 \mathrm{~cm}^{2}$, these power densities can be achieved at moderate current levels.

Since the etch rate is linearly related to electrode power density, it is worthwhile to evaluate the etch rate per unit power density as a figure of merit for the efficiency of the etch. Figure 8 shows this parameter as a function of chamber pressure for three minute long etches performed though a $280 \mu \mathrm{m}$ wide slit opening in titanium electrodes. As the pressure is increased, the power necessary to sustain the plasma increases from 3-7 W/ $\mathrm{cm}^{2}$. Despite this, the ratio of the etch rate to power density increases from 0.15 to

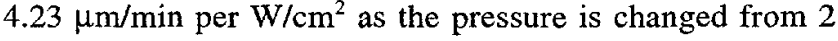
to 20 Torr. Higher ratios achieved by other operating parameters are also shown in Fig. 8.

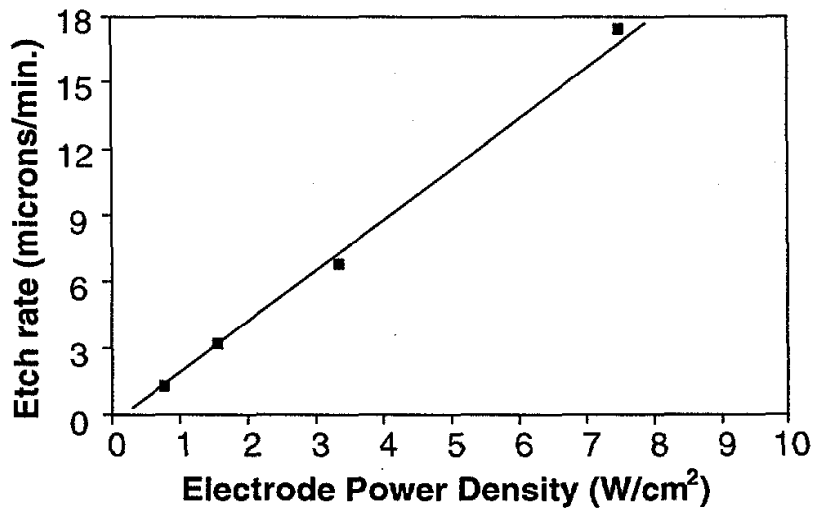

Fig 7: The etch rate is proportional to electrode power density. (All 3 min. etches at 2.7 Torr.)

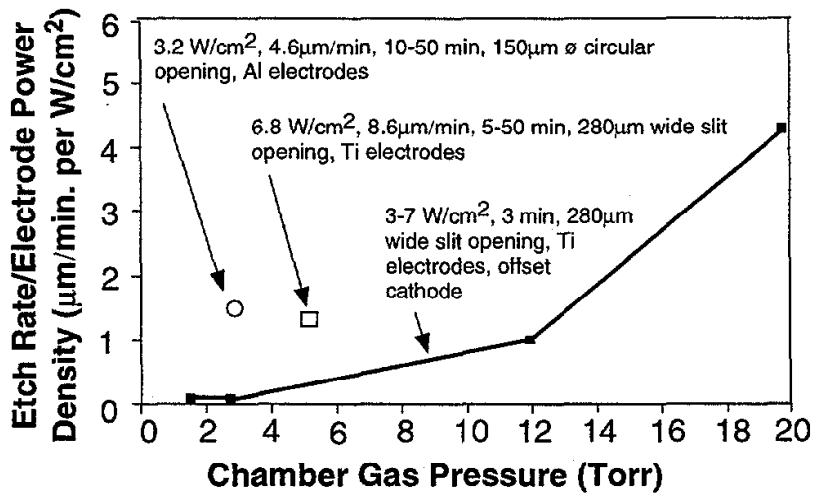

Fig 8: The ratio of the etch rate to the electrode power density increases with chamber pressure. 


\section{PLASMA MODELING}

The resistivity of a conventional plasma can be estimated by [4]:

$$
\eta \approx \frac{\pi q^{2} \sqrt{m}}{\left(4 \pi \varepsilon_{o}\right)^{2}(k T)^{3 / 2}} \ln \Lambda ; \quad \Lambda=\overline{L_{D} / r_{o}} ; \quad r_{o}=\frac{q^{2}}{4 \pi \varepsilon_{o} m v^{2}}
$$

where $\varepsilon_{0}$ is the permittivity of free space, $k$ it the Boltzmann constant, $T$ is the electron temperature, $q$ is the electron charge, $m$ is electron mass, and $v$ is electron velocity. For laboratory plasmas, $\ln \Lambda$ is close to 10 . To the extent that the electrode power density is linearly related to $k T$, if the plasma dimensions remain relatively constant, eqn. (1) suggests that the plasma resistance should be proportional to $P^{-3 / 2}$, where $P$ denotes the power density. Figure 9 plots the measured resistance of the microplasmas as a function of $P$. It also shows the best fit of these points to a curve proportional to $P^{-3 / 2}$, which is achieved using a proportionality constant of 2.2. The measurements for Fig. 9 were obtained at 2.7 Torr using aluminum electrodes of $0.2 \mathrm{~cm}^{2}$ area.

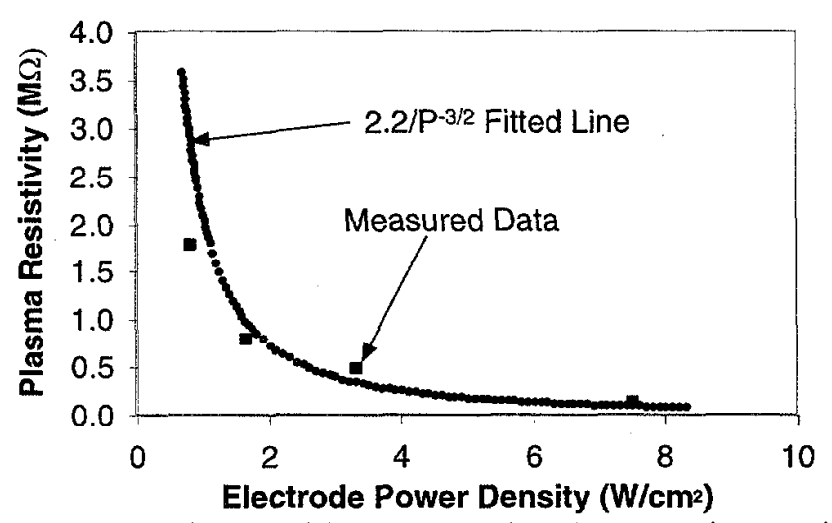

Fig 9: Consistent with eqn. (1), the plasma resistance is proportional to $P^{-3 / 2}$, where $P$ is the power density.

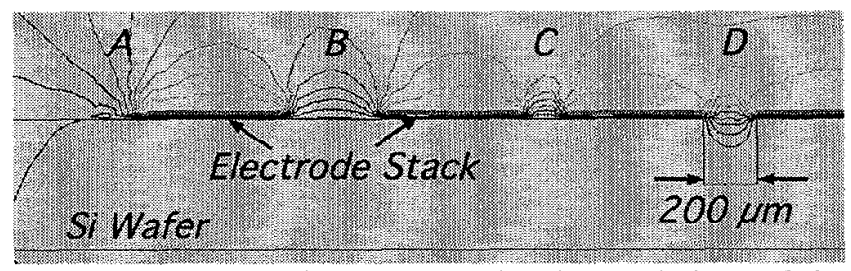

Fig. 10: Equipotential contours showing variations of the local electric field with mask openings of varying widths $(A-C)$ and with etch progression $(D)$.

Under the etching conditions described in the preceding section, the etching is believed to be performed by ions which are pulled away from the sheath that exists above the electrode stack by the electric field associated with the openings in the stack. Results from a preliminary effort at modeling the electric field are shown in Fig. 10. The simulations were performed using MAXWELL ${ }^{\mathrm{TM}}$, and neglect the conductivity and charge distribution of the plasma. The geometry assumes openings of various widths in a $30 \mu \mathrm{m}$ thick polyimide-metal-polyimide electrode stack on a $500 \mu \mathrm{m}$ thick silicon wafer. The relative dielectric constant of the polyimide is 3.5 , and the resistivity of the metal is zero. The figure shows equipotential contours which are crowded progressively closer together in openings $A, B$, and $\mathrm{C}$, suggesting that the electric fields are higher above narrow openings. At the same time, the local electric field extends to greater heights above the wider gaps. The difference between the contours at locations $C$ and $D$, which are of the same width but represent shallow and deep etches, respectively, suggest that the electric field is reduced as an etch progresses. This may be correlated to the observation made in the preceding section that the initial etch rate is higher than the cumulative average rate.

\section{CONCLUSIONS}

This effort has demonstrated the use of microplasmas for the etching of silicon. The microplasmas were generated by DC power applied to in-situ electrodes patterned on the silicon wafer that also served as an etch mask and a shield against the applied potential. Short etches at rates exceeding $17 \mu \mathrm{m} / \mathrm{min}$. and sustained etch rates in the range of $4-10 \mu \mathrm{m} / \mathrm{min}$. were demonstrated, and through-wafer etches were achieved using $\mathrm{SF}_{6}$. The typical operating pressure and power density were in the range of 1-20 Torr and $1-10 \mathrm{~W} / \mathrm{cm}^{2}$, respectively. Varying degrees of anisotropy were achieved by changing the etch conditions. The etch rate depended linearly on the electrode power density; the power efficiency of the etch rate increased with pressure. The change of the plasma resistance with the power density was found to comply with theoretical expectations.

\section{ACKNOWLEDGEMENTS}

The authors thank Mr. Mo-Huang Li for help with sample preparation.

\section{REFERENCES}

[1] W.H. Juan, S.W. Pang, "Released Si Microstructures Fabricated by Deep Etching and Shallow Diffusion," IEEE J. Microelectromechanical Sys., 5(1), March 1996, pp. 18-23

[2] C. Marxer, C. Thio, M.-A. Gretillat, N.F. de Rooij, R. Battig, O. Anthmatten, B. Valk, P. Vogel, "Vertical Mirrors Fabricated by Deep Reactive Ion Etching for Fiber-Optic Switching Applications," IEEE J. Microelectromechanical Sys., 6(3), Sep. 1996, pp. $227-$ 285

[3] W.-H. Juan, S.W, Pang, A. Selvakumar, M.W. Putty, K. Najafi, "Using Electron Cyclotron Resonance (ECR) Source to Etch Polyimide Molds for Fabrication of Electroplated Microstructures," Proc., Solid-State Sensors and Actuators Workshop, Hilton Head, SC, June 1994, pp. 82-85

[4] F.F. Chen, Introduction to Plasma Physics and Controlled Fusion, Plenum Press, 1983, pp. 181 